\title{
EXTRACTION OF SYMBOLIC STATEMENTS FROM SYNAPTIC WEIGHTS
}

\author{
W.J. Daunicht, R. Steiner, and H. Franz
}

Neurologisches Therapiecentrum, Hohensandweg 37, D-4000 Düsseldorf, Germany

In general, neural networks are 'opaque', i.e. it seems impossible to understand the meaning of their adaptive parameters such as synaptic weights. The difficulty to extract symbolic information or derive rules about the internal structure of a problem by interpretation of the synaptic weights after training is one of the fundamental problems in neural network theory.

The DEFAnet concept $[1,2]$, however, lends itself to such an analysis. A DEFAnet is a 3-layered feedforward network with $n$ inputs where the input space is subdivided into an $n$-dimensional grid in the first layer. The outputs of the second layer form polynomials of first order within a grid cell. The output layer of the network is fully connected to the neurons of the second layer forming scalar products of the weights and the output of these neurons. Only these synapses are plastic and can be determined by calculation or training. Then, they can be used to derive symbolic information about the internal structure of the network function.

For each pair of inputs it is tested, whether all the synaptic weights corresponding to a product of the pair is smaller than a small positive $\epsilon$. If so an additive relation between the two inputs has been detected and the network function can be considered as the sum of two-possibly nonlinear functions of one of the inputs, as long as the rest of the arguments is constant. Thus, the desired function can be interpreted as the sum of the outputs of two separate networks, each omitting one of the inputs of the pair. A positive result of this test can be entered as 1 , in a symmetric $n \times n$ incidence matrix $M$ holding the relations between all possible pairs of inputs. If a row or column (except the diagonal element) is filled with 1s only, the contribution of that single input can be identified as a summand to the rest of the function.

Furthermore, the linearity of the function in an input can be tested. For this analysis, each input interval in the network used must be subdivided into at least 2 subintervals. Then, the synaptic weights corresponding to products differing only in the subinterval of the particular input are analysed. If all these weights differ by less than $\epsilon$, the network function is linear in this input and the corresponding diagonal element of $\mathbf{M}$ is set to 1 . If the weights are small themselves, the input does not contribute to the network function at all and the appropriate diagonal matrix element is set to -1 ; otherwise it remains 0 .

Some special cases of the matrix $M$ can be easily interpreted as shown in the table where $f$ is the network function, $f^{*}$ is a general nonlinear function, and $a, b, c$ are parameters.

The results can be used to tailor a DEFAnet in such a way as to minimize both network size and training time. In case of additivity between two inputs the corresponding

\begin{tabular}{|l|l|}
\hline Matrix M & Statement \\
\hline $\mathbf{M}=\mathbf{0}$ & $f(\underline{x})=f^{*}(\underline{x})$ \\
$\mathbf{M}=1$ & $f(\underline{x})=\sum_{\nu=1}^{n} a_{\nu} x_{\nu}+c$ \\
$\mathbf{M}=\mathbf{I}$ & $f(\underline{x})=\prod_{\nu=1}^{n}\left(a_{\nu} x_{\nu}+b_{\nu}\right)$ \\
$\mathbf{M}=1-\mathbf{I}$ & $f(\underline{x})=\sum_{\nu=1}^{n} f_{\nu}^{*}\left(x_{n}\right)$ \\
$\mathbf{M}=1-2 \mathrm{I}$ & $f(\underline{x})=c$ \\
\hline
\end{tabular}
second layer neurons can be omitted. In case of linearity, those neurons in the first layer covering the linear range can be replaced by single neurons thereby reducing the number of second layer neurons and plastic synapses.

In addition, the results of that analysis can be used for the evaluation of the influence of certain inputs and for the detection of some internal structure of an unknown function. It may also help to uncover architectures of unknown nonlinear systems or may detect regularities in discrete data. It is concluded, that the DEFAnet concept and the extraction of information from synaptic weights can be a useful tool for the analysis of unknown functions.

\section{References}

[1] W.J. Daunicht. DEFAnet - a deterministic neural network concept for function approximation. Neural Networks, 4:839-845, 1991.

[2] W.J. Daunicht. DEFAnet2 - advancements of a deterministic function approximator. In this conference, 1993.

Acknowledgements: This work was supported by DFG grant Da 199/2-1. 